\title{
In vitro endothelial hyperpermeability occurs early following traumatic hemorrhagic shock
}

\author{
Anoek L.I. van Leeuwen ${ }^{\mathrm{a}, \mathrm{b}}$, David N. Naumann ${ }^{\mathrm{c}, \mathrm{d}}$, Nicole A.M. Dekker ${ }^{\mathrm{a}, \mathrm{b}}$, \\ Peter L. Hordijk ${ }^{\mathrm{b}}$, Sam D. Hutchings ${ }^{\mathrm{d}, \mathrm{e}}$, Christa Boer ${ }^{\mathrm{a}}$ and Charissa E. van den Brom ${ }^{\mathrm{a}, \mathrm{b}, *}$ \\ ${ }^{a}$ Department of Anesthesiology, Experimental Laboratory for VItal Signs, Amsterdam Cardiovascular \\ Sciences, Amsterdam UMC, VU University, Amsterdam, The Netherlands \\ ${ }^{\mathrm{b}}$ Department of Physiology, Experimental Laboratory for VItal Signs, Amsterdam Cardiovascular \\ Sciences, Amsterdam UMC, VU University, Amsterdam, The Netherlands \\ ${ }^{\mathrm{c} S u r g i c a l}$ Reconstruction and Microbiology Research Centre, University Hospitals Birmingham, \\ Queen Elizabeth Hospital, Birmingham, UK \\ ${ }^{\mathrm{d}}$ Academic Department of Military Anesthesia and Critical Care, Royal Centre for Defense Medicine, \\ Birmingham, UK \\ ${ }^{\mathrm{e}}$ Department of Critical Care, King's College Hospital, London, UK
}

\begin{abstract}
.
BACKGROUND: Endothelial hyperpermeability is suggested to play a role in the development of microcirculatory perfusion disturbances and organ failure following hemorrhagic shock, but evidence is limited.

OBJECTIVE: To study the effect of plasma from traumatic hemorrhagic shock patients on in vitro endothelial barrier function.

METHODS: Plasma from traumatic hemorrhagic shock patients was obtained at the emergency department (ED), the intensive care unit (ICU), $24 \mathrm{~h}$ after ICU admission and from controls $(n=8)$. Sublingual microcirculatory perfusion was measured using incident dark field videomicroscopy at matching time points. Using electric cell-substrate impedance sensing, the effects of plasma exposure on in vitro endothelial barrier function of human endothelial cells were assessed.

RESULTS: Plasma from traumatic hemorrhagic shock patients collected at ED admission induced a $19 \%$ loss of in vitro endothelial resistance compared to plasma from controls $(p<0.001)$. This loss was due to reduced cell-cell contacts $(p<0.01)$. Plasma withdrawn at later time points did not affect endothelial barrier function $(p>0.99)$. Interestingly, in vitro endothelial resistance showed a positive association with in vivo microcirculatory perfusion $(r=0.56, p<0.01)$.

CONCLUSIONS: Plasma from traumatic hemorrhagic shock patients obtained following ED admission, but not at later stages, induced in vitro endothelial hyperpermeability. This coincided with in vivo microcirculatory perfusion disturbances.
\end{abstract}

Keywords: Hemorrhagic shock, endothelial barrier function, microcirculation, plasma, endothelial permeability

\section{Introduction}

The endothelial barrier holds a key position in the regulation of paracellular and transcellular transportation of liquids and solutes due to its semi-permeable properties [1]. Under normal conditions, endothelial cells are tightly bound and permeability is limited to extravasation of water and electrolytes.

\footnotetext{
*Corresponding author: Charissa van den Brom, PhD, Department of Anesthesiology, Amsterdam UMC - VU University medical center, De Boelelaan 1117, 1081 HV Amsterdam, The Netherlands. Tel.: +31 20 4441433; Fax: +31 20 4444385; E-mail: c.vandenbrom@amsterdamumc.nl.
} 
However, as a result of a pathological insult, permeability increases and leads to extravascular leakage of macromolecules with edema formation as a consequence [1].

Circulatory shock is one of the pathological conditions leading to endothelial hyperpermeability, and is defined as a state of acute circulatory failure leading to decreased tissue oxygen delivery [2]. Based on the underlying cause, shock can be characterized as cardiogenic, obstructive, disruptive and hypovolemic [2]. Hemorrhagic shock, a type of hypovolemic shock, is known to induce edema formation, microcirculatory perfusion disturbances [3,4] and multiple organ failure in patients [4]. Hemorrhagic shock is accountable for $21 \%$ to $39 \%$ of deaths following trauma, of which the majority occurs in the pre-hospital phase [5]. Little is however known regarding the exact mechanism causing edema formation and microcirculatory perfusion disturbances following hemorrhagic shock, hampering the development of potential treatment targets and strategies.

Most of our understanding regarding hemorrhagic shock-induced endothelial hyperpermeability is derived from animal studies or from the evaluation of plasma markers involved in inflammation and endothelial activation and injury. Inflammation, glycocalyx degradation, mitochondrial dysfunction and disruption of endothelial junctions are mechanisms contributing to increased endothelial permeability [6, 7]. However, the direct effect of plasma from patients following traumatic hemorrhagic shock on endothelial permeability and its course remains unknown.

Therefore, we investigated the effect of plasma collected from patients following traumatic hemorrhagic shock over time on endothelial permeability using an in vitro bioassay for endothelial barrier function. We subsequently studied its relation to in vivo microcirculatory perfusion and circulating markers known to be associated with endothelial injury and permeability.

\section{Materials and methods}

\subsection{Study protocol}

The Microshock study was approved in the UK under Research Ethics Committees reference: 14/YH/0078 (Clinical trial registration: NCT02111109) [8]. The current study includes a subgroup analysis of 16 adult patients from a single site (Birmingham, UK) [8]. These patients had evidence of hemorrhagic shock as represented by a mechanism of injury consistent with blood loss. Eligible patients needed to be intubated and ventilated, have a serum lactate concentration $>2 \mathrm{mmol} . \mathrm{l}^{-1}$ recorded at any stage prior to admission to the intensive care unit (ICU), and received any blood products (e.g., packed red blood cells (PRBC), fresh frozen plasma (FFP), cryoprecipitate) during the initial period of resuscitation, prior to admission to the ICU. A process of deferred consent was approved by the research ethics committee [8]. Characteristics and serum analyses of syndecan-1 and soluble thrombomodulin $[9,10]$ and microcirculatory perfusion measurements of these patients [4] were published previously.

\subsection{Blood sampling}

Blood was collected in both citrate and z-serum clotting activator tubes (for plasma and serum samples respectively) at three different time points, which include arrival at the emergency department (ED), admission at the ICU (ICU) and 24 hours after admission at the ICU (ICU+24 h) (Fig. 1A). To reach $n=8$ per time point as derived by our power calculation, plasma was withdrawn from 16 patients in total as it was difficult to include plasma at three time points from the same patient. Eight patients had available blood samples at two time points, the remaining patients had an available blood sample at one time point. Plasma was also collected from eight age- and sex-matched controls. For serum samples, blood from z-serum clotting activator tubes was centrifuged at $1,620 \mathrm{G}$ for $10 \mathrm{~min}$ at $4^{\circ} \mathrm{C}$. For 


\section{A. Study protocol}

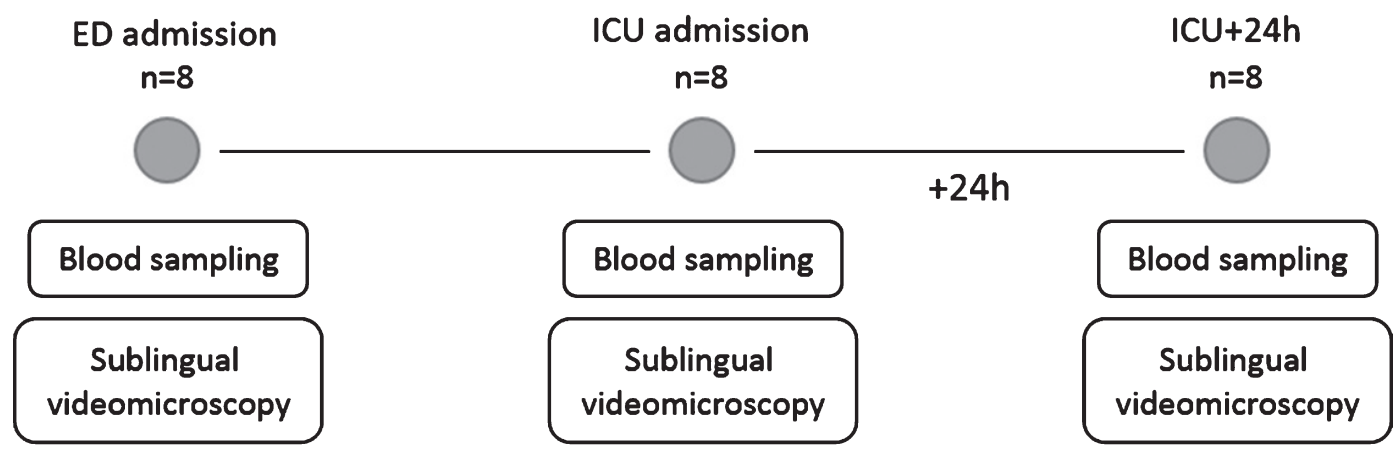

\section{B. In vitro experimental set-up}

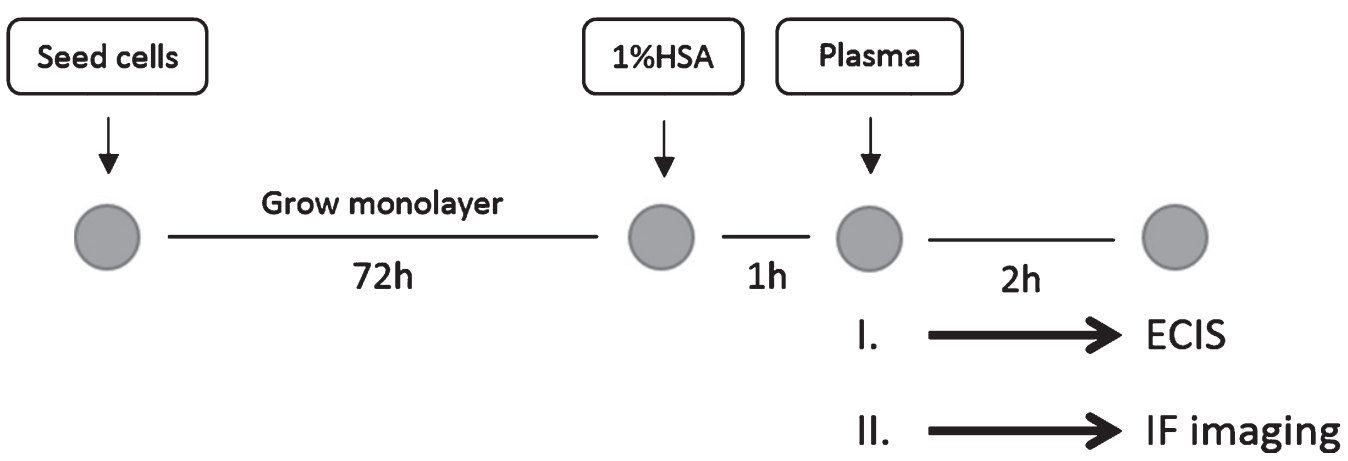

Fig. 1. Study protocol and in vitro experimental set-up. (A) Blood was collected (for plasma and serum samples respectively) and sublingual non-invasive incident dark field videomicroscopy was performed at three different time points: after arrival at the emergency department (ED; $n=8$ ), after admission at the intensive care unit (ICU; $n=8$ ) and 24 hours later (ICU+24 h; $n=8$ ). (B) Human endothelial cells were seeded and culture until a monolayer was formed. After starvation in $1 \%$ human serum albumin (HSA), endothelial cells were exposed to plasma from traumatic hemorrhagic shock patients collected at ED admission, ICU admission or $24 \mathrm{~h}$ later. Measurements were performed using electric cell-substrate impedance sensing (ECIS; experiment I.) or via immunofluorescence imaging (IF imaging; experiment II.).

plasma samples, blood from citrate tubes was centrifuged at $2,000 \mathrm{G}$ for $20 \mathrm{~min}$ at $4{ }^{\circ} \mathrm{C}$, and then the supernatant was additionally centrifuged at $13,000 \mathrm{G}$ for $2 \mathrm{~min}$ at $4^{\circ} \mathrm{C}$ to achieve platelet-free plasma. The platelet-free plasma and serum samples were stored at $-80^{\circ} \mathrm{C}$.

\subsection{Cell culture}

For cell culture experiments, the following materials were used: bare medium (bM199) consisting of Medium 199 supplemented with penicillin $100 \mathrm{U} \cdot \mathrm{ml}^{-1}$ and streptomycin $100 \mathrm{mg} \cdot \mathrm{ml}^{-1}$ (all from Biowhittaker, Verviers, Belgium); complete medium (cM199) consisting of bM199 supplemented with $10 \%$ heat inactivated new-born calf serum (Gibco, Grand Island, NY, USA), $10 \%$ heat inactivated human serum (pooled serum of 10-20 healthy donors, stored at $4^{\circ} \mathrm{C}$, Sanquin CLB, Amsterdam,

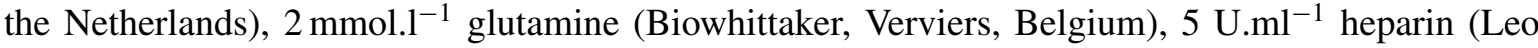
Pharmaceutical Products, Weesp, The Netherlands), $150 \mu \mathrm{g} \cdot \mathrm{ml}^{-1}$ crude endothelial cell growth factor prepared from bovine hypothalamus; $1 \%$ HSA solution (dilution of human serum albumin in bM199; Sanquin, Amsterdam, the Netherlands) and Trypsin (Gibco, Grand Island, NY, USA). 
Human umbilical vein endothelial cells (HUVECs) were isolated and cultured as described before $[11,12]$. Briefly, endothelial cells were isolated from human umbilical cords obtained from healthy donors from Amstelland Hospital (Amstelveen, the Netherlands) and subsequently cultured on gelatincoated well plates in cM199 medium at $37^{\circ} \mathrm{C}$, in an atmosphere of $95 \%$ air and $5 \% \mathrm{CO}_{2}$.

\subsection{Electric cell-substrate impedance sensing}

Electric cell-substrate impedance sensing (ECIS; Applied BioPhysics, Troy, NY, USA) was used to measure impedance of endothelial cells as previously described [13]. Confluent, passage one HUVECs pooled from three donors were transferred to gelatin-coated 96-wells ECIS culture plates (Applied BioPhysics, Troy, NY, USA) pre-treated with $10 \mathrm{mM}$ L-cysteine (Merck, Darmstadt, Germany). After $72 \mathrm{~h}$ of culturing in cM199 medium, the ECIS device was used for continuous, multi frequency scanning to confirm a confluent monolayer. Confluent monolayers were washed with and exposed to $1 \%$ human serum albumin (HSA) in bare medium for 60 min followed by addition of 10\% platelet-free plasma collected from traumatic hemorrhagic shock patients or age- and sex-matched controls. Impedance was recorded for 2 hours until steady state was reached at multiple frequencies ranging from $250-64000 \mathrm{~Hz}$ using ECIS software (v1.2.210.0 PC; Applied Bio-Physics). A schematic overview of the experimental set-up is shown in Fig. 1B. Plasma concentration and exposure time were based on previously performed experiments [12] and confirmed in pilot experiments.

Resistance is one of the parameters that is derived from impedance measurements and represents quality and function of the cell barrier [13]. Impedance was also used to calculate cell-cell integrity $(\mathrm{Rb})$ and cell-matrix integrity $(\alpha)$. All measurements were performed in triplicate and normalized to baseline.

\subsection{Immunofluorescence imaging}

Immunofluorescence was used to visualize cell structures of interest. HUVECs were seeded on $5 \mu \mathrm{M}$ fibronectin-coated coverslips. After reaching a confluent monolayer, cells were washed with and exposed to $1 \%$ HSA for 60 min followed by 2 hour stimulation with $10 \%$ platelet-free plasma from either traumatic hemorrhagic shock patients collected upon arrival at the $\operatorname{ED}(n=5)$ or age- and sexmatched controls $(n=8)$ (Fig. 1B). Cells were washed with bM199 and fixed with 4\% paraformaldehyde at room temperature. Upon fixation, cells were permeabilized with $0.2 \%$ Triton X-100 in PBS for 3 min and unspecific staining was blocked by incubation with $1 \%$ HSA. Cells were incubated with a primary antibody against VE cadherin (C-19, 1:400, Santa Cruz, Dallas, TX, USA) and a secondary antibody against donkey anti-goat Alexa Fluor 488 (Invitrogen, Carlsbad, CA, USA). DAPI (Thermo Fisher Scientific, Waltham, MA, USA) and acti-stain phalloidin 670 (Cytoskeleton, Denver, CO, USA) were used to stain nuclei and F-actin, respectively. Coverslips were mounted in Mowiol mounting solution (Sigma Aldrich, Zwijndrecht, The Netherlands). Imaging was performed with a Zeiss Axiovert 200M Marianas $^{\mathrm{TM}}$ digital imaging inverted microscope system using a $63 x$ Zeiss oil objective (Zeiss, Breda, the Netherlands).

\subsection{Microcirculatory perfusion measurements}

Microcirculatory perfusion has previously been reported for 58 patients included in the Microshock study [4]. Briefly, sublingual microcirculatory perfusion was measured using non-invasive incident dark field (IDF) videomicroscopy (Cytocam, Braedius Medical, Huizen, the Netherlands). In vivo microcirculatory perfusion measurements were performed at time points corresponding to blood sampling (Fig. 1A). At least four video clips of 5 seconds (100 frames) duration were recorded and stored 
de-identified for blinded analysis. Microcirculatory videos were analyzed by a single operator using automated vascular analysis (AVA) v.3.02 (Microvision Medical, Amsterdam, The Netherlands) by manually tracing all vessels by hand. These video analyses give values for total vessel density (TVD), perfused vessel density (PVD) and proportion of perfused vessels (PPV) according to the current best practice guidelines for reporting microcirculatory variables [4]. Here, in vivo microcirculatory perfusion measurements of the 16 patients that were included in the present study were used for additional analysis.

\subsection{Serum analysis}

Serum concentrations of angiopoietin-1, angiopoietin-2, soluble Tie2 (R\&D Systems, Minneapolis, MN, USA) and von Willebrand factor (Abcam, Cambridge, MA) were analyzed using commercially available enzyme-linked immunosorbent assays. Serum levels of both syndecan-1 and soluble thrombomodulin have previously been reported of the patients in this cohort $[9,10]$, and were included in additional analyses in the current study.

\subsection{Statistical analysis}

Data were analyzed using Graphpad Prism 7.0 (Graphpad Software, La Jolla, CA, USA).

At least a $20 \%$ reduction $(\Delta=230 \mathrm{Ohm})$ in in vitro endothelial resistance with a standard deviation of $140 \mathrm{Ohm}$ following exposure to plasma of traumatic hemorrhagic shock patients was expected [12]. With a significance level $(\alpha)$ of 0.05 and beta of 0.9 group sizes of $n=8$ were calculated.

Continuous data are expressed as mean \pm standard deviation for normally distributed data, or median followed by the interquartile range (Q1-Q3) for non-normal data. Normality of distribution was tested with the Shapiro-Wilk test. Changes in endothelial resistance over time were evaluated using twoway ANOVA with Bonferroni post-hoc analyses. An one-way ANOVA with Bonferroni post-hoc analysis or Kruskal Wallis test was used to evaluate differences between groups for normally and nonnormally distributed data, respectively. Associations between in vitro endothelial resistance with patient characteristics and microcirculatory perfusion parameters were analyzed using Pearson's correlation coefficient or Spearman's rank correlation coefficient for normally and non-normally distributed data, respectively. $P$ values $<0.05$ were considered statistically significant.

\section{Results}

\subsection{Patient characteristics}

Sixteen traumatic hemorrhagic shock patients enrolled in the Microshock study with available blood samples were included. Patient characteristics and clinical parameters are listed in Table 1. Patients had a median age of $38(23-53)$ years, were mostly male (94\%) and had an injury severity score (ISS) of 27 (15 - 34), lowest systolic blood pressure of $94(62-109) \mathrm{mmHg}$ and highest lactate level of 5.9

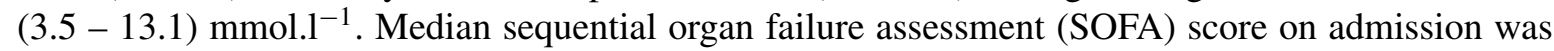
10 (6 - 12). Median time between ED admission and ICU admission was $4(2-8) \mathrm{h}$, median hospital stay was $18(6-42)$ days and mortality rate was $25 \%$. 

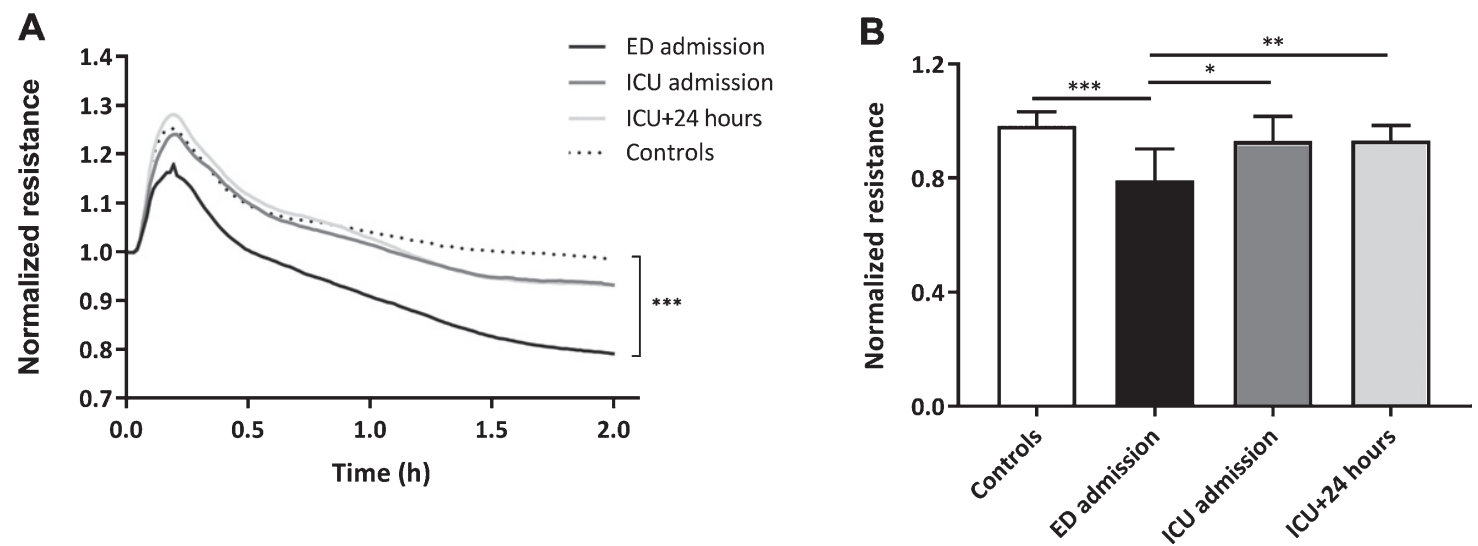

Fig. 2. Loss of in vitro endothelial barrier function. Human endothelial cells were exposed to plasma from traumatic hemorrhagic shock patients collected at admission at the emergency department (ED), intensive care unit (ICU), 24 hours (ICU+24 h) after admission at the ICU and from controls (all $n=8)$. Endothelial resistance after plasma exposure over time (A) and after 2 hours of plasma exposure (B). A: Data represent mean and were tested with a two-way ANOVA with Bonferroni post-hoc analysis. B: Data represent mean $\pm \mathrm{SD}$ and were tested with a one-way ANOVA with Bonferroni post-hoc analysis. ${ }^{*} p<0.05$, ${ }^{* *} p<0.01,{ }^{* * *} p<0.001$ compared to ED admission.

\subsection{Plasma from traumatic hemorrhagic shock patients induced endothelial hyperpermeability}

Plasma obtained directly at ED admission decreased in vitro endothelial resistance with $19 \%$ compared to plasma from controls ( $p<0.001$, Fig. 2A and B). This reduction in in vitro endothelial resistance was absent following exposure to plasma obtained at ICU admission and 24 hours later compared to controls (both $p>0.99$ ). Compared to ED admission, plasma collected at ICU admission and 24 hours later increased endothelial resistance ( $p<0.05, p<0.01$, Fig. $2 \mathrm{~A}$ and B).

Patients following traumatic hemorrhagic shock showed a decrease in hematocrit over time (Table 1). No association was found between hematocrit and in vitro endothelial resistance $(r=-0.33, p=0.11)$.

\subsection{Plasma from traumatic hemorrhagic shock patients impaired endothelial cell-cell integrity}

Two parameters can be modelled from endothelial resistance measurements, which distinguish between cell-cell $(\mathrm{Rb})$ and cell-matrix (alpha) integrity (Fig. 3A). Plasma collected at ED admission decreased cell-cell integrity by $35 \%$ compared to plasma from controls $(p<0.01)$, but no differences were measured between the remaining time points and controls (Fig. 3B). No differences in cell-matrix integrity were found between all time points (Fig. 3C).

Increased intercellular gap formation was observed using immunofluorescence staining of human endothelial cells exposed to plasma from traumatic hemorrhagic shock patients collected at ED admission compared to plasma from controls, which was paralleled by a reorganization of actin filaments from cortical actin distribution to fibers that stretch throughout the endothelial cell body, indicating increased stress-fiber formation (Fig. 3D).

\subsection{Microcirculatory perfusion and in vitro endothelial barrier function}

In vitro endothelial resistance was positively associated with in vivo microcirculatory perfusion, as represented by the proportion of perfused vessels and perfused vessel density (PPV: $r=0.56, p<0.01$, 
Table 1

Patient characteristics

\begin{tabular}{|c|c|}
\hline & Patients $(n=16)$ \\
\hline Age (years) & $38(23-53)$ \\
\hline Men & $15(94 \%)$ \\
\hline Injury Severity Score & $27(15-34)$ \\
\hline \multicolumn{2}{|l|}{ SOFA score } \\
\hline ED admission & $10(6-12)$ \\
\hline 7 days after admission & $5(2-21)$ \\
\hline \multicolumn{2}{|l|}{ Macrocirculatory variables } \\
\hline Lowest systolic blood pressure (mmHg) & $94(62-109)$ \\
\hline \multicolumn{2}{|l|}{ Hematocrit $\left(1.1^{-1}\right)$} \\
\hline ED admission & $0.37(0.32-0.41)$ \\
\hline ICU admission & $0.29(0.27-0.34)$ \\
\hline$I C U+24 h$ & $0.28(0.24-0.36)^{*}$ \\
\hline \multicolumn{2}{|l|}{ Lactate $\left(\mathrm{mmol} . \mathrm{l}^{-1}\right)$} \\
\hline ED admission & $5.4(2.3-16.1)$ \\
\hline ICU admission & $3.0(1.7-4.0)$ \\
\hline$I C U+24 h$ & $1.5(1.1-3.5)^{*}$ \\
\hline \multicolumn{2}{|l|}{ Base excess; $\left(\mathrm{mEq} .1^{-1}\right)$} \\
\hline ED admission & $-8.6(-21.3--2.4)$ \\
\hline ICU admission & $-3.0(-5.8--0.4)$ \\
\hline$I C U+24 h$ & $-2.7(-4.7-2.1)$ \\
\hline \multicolumn{2}{|l|}{ Type of trauma } \\
\hline Penetrating $(n)$ & $4(25 \%)$ \\
\hline Blunt $(n)$ & $12(75 \%)$ \\
\hline \multicolumn{2}{|l|}{ Clinical outcomes } \\
\hline Hospital stay (days) & $18(6-42)$ \\
\hline Mortality rate; $(n)$ & $4(25 \%)$ \\
\hline \multicolumn{2}{|l|}{ Resuscitation fluids in first $24 \mathrm{~h}$} \\
\hline Packed RBCs (units) & $4(3-6)$ \\
\hline Fresh frozen plasma (units) & $2(1-4)$ \\
\hline Crystalloids (1) & $4.4(2.7-5.7)$ \\
\hline Colloids (1) & $0(0-0)$ \\
\hline Noradrenaline dose $\left(\mu \mathrm{g} . \mathrm{kg} \cdot \mathrm{min}^{-1}\right)$ & $0.22(0.11-0.58)$ \\
\hline
\end{tabular}

Fig. 4 and PVD: $r=0.38, p=0.07$ ), but not total vessel density (TVD: $r=0.08, p=0.73$ ), measured at matching time points.

\subsection{Early changes in circulating markers and in vitro endothelial barrier function}

Patients showed an increase over time in circulating angiopoietin-2 $(p<0.01)$, whereas circulating angiopoietin-1, soluble Tie 2 and von Willebrand factor levels remained unchanged (Table 2). Circulating levels of syndecan-1 and soluble thrombomodulin have previously been reported [9, 10]. Similar results were found if circulating markers were corrected for hematocrit (data not shown). No associa- 
A

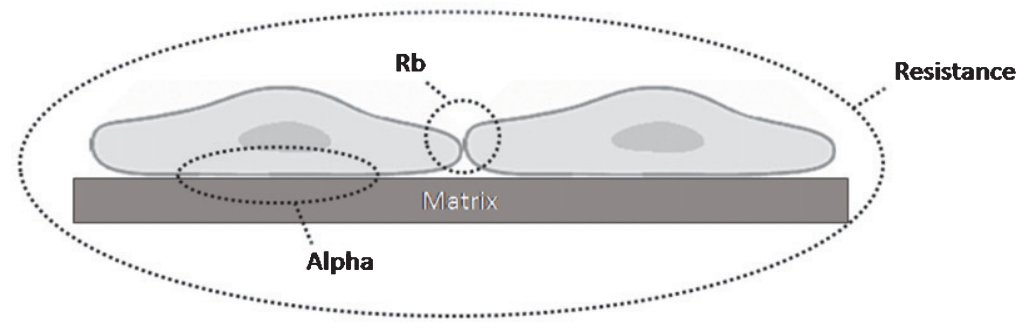

B

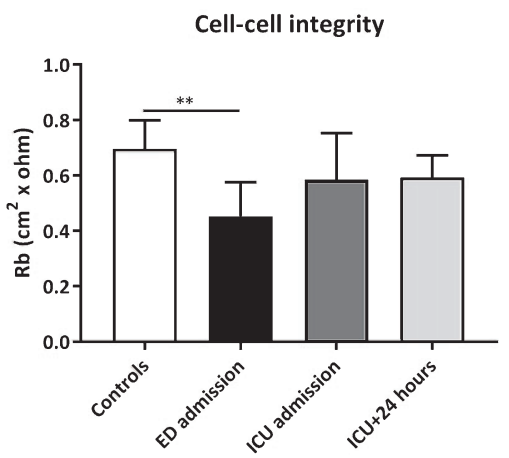

C

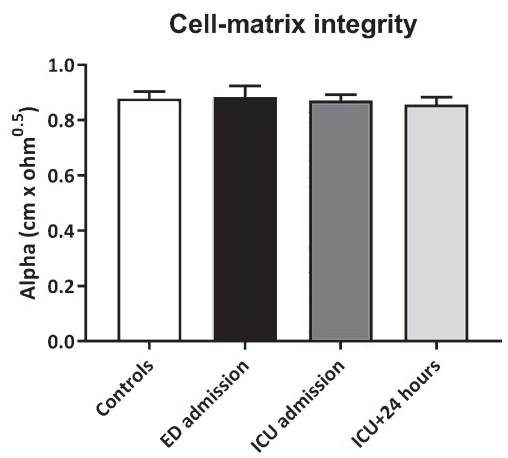

D
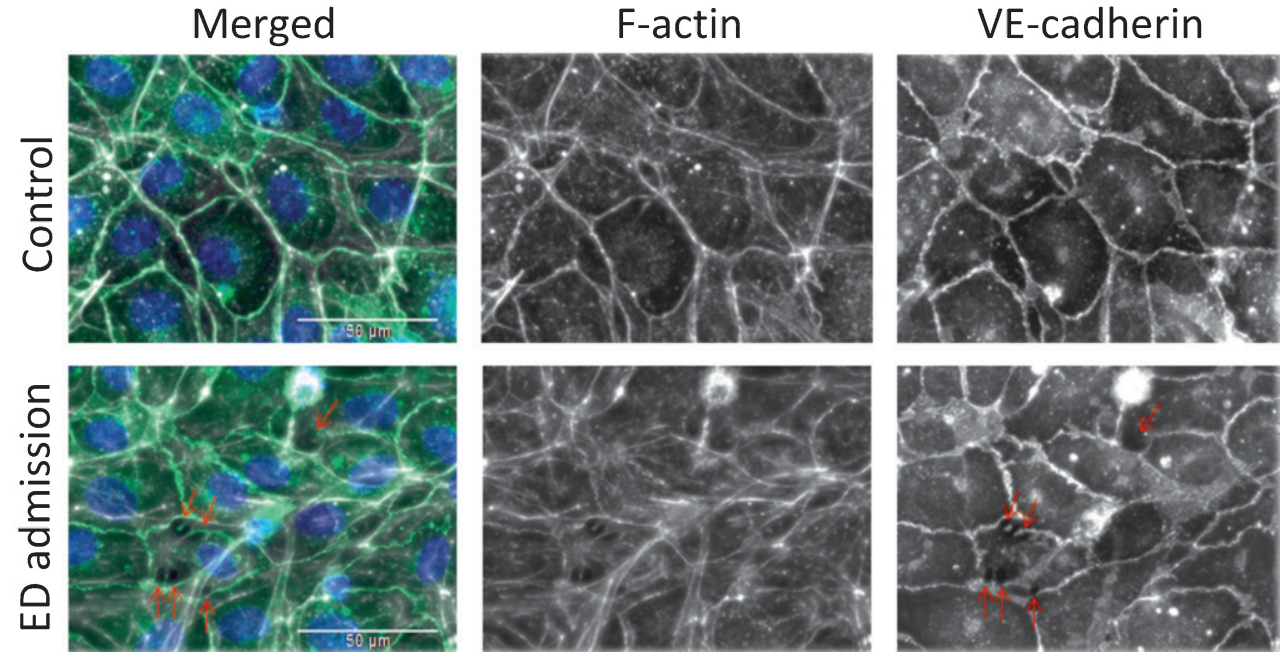

Fig. 3. Impaired in vitro endothelial cell-cell integrity. Rb and alpha can be modelled from endothelial resistance measurements, which distinguish between cell-cell $(\mathrm{Rb})$ and cell-matrix (alpha) integrity (A). Human endothelial cells were exposed to plasma from traumatic hemorrhagic shock patients collected at admission at the emergency department (ED), intensive care unit (ICU), 24 hours (ICU $+24 \mathrm{~h})$ after admission at the ICU and from controls (all $n=8)$. Cell-cell integrity (Rb; B), cell-matrix integrity (alpha; C) and representative images of endothelial cells stained for VE cadherin (adherents junctions; green), F-actin (stress fibers; white) and DAPI (nuclei; blue) (D) after 2 hours of plasma exposure. Data represent mean \pm SD and were tested with a one-way ANOVA with Bonferroni post-hoc analysis, ${ }^{* *} p<0.01$ compared to ED admission. 


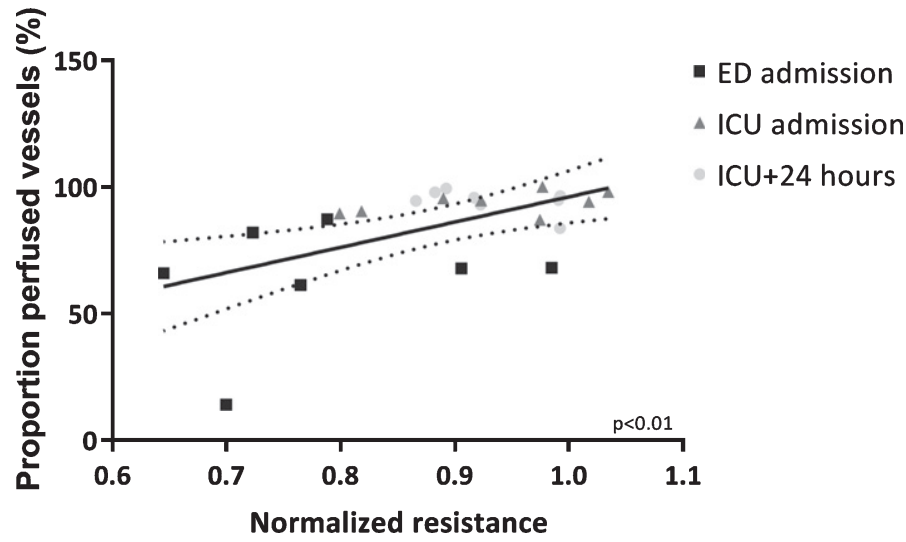

Fig. 4. Microcirculatory perfusion associated with in vitro endothelial barrier function. Association between proportion of perfused vessels and in vitro endothelial resistance determined after exposure to plasma collected at corresponding time points. Data are presented with a linear regression with 95\% CI and tested with a Pearson's correlation test.

Table 2

Circulating serum markers

\begin{tabular}{lcccc}
\hline & $\begin{array}{c}\text { ED admission } \\
(n=8)\end{array}$ & $\begin{array}{c}\text { ICU admission } \\
(n=8)\end{array}$ & $\begin{array}{c}\text { ICU+24h } \\
(n=8)\end{array}$ & $p$-value \\
\hline Angiopoietin-1 $\left(\mathrm{ng} . \mathrm{ml}^{-1}\right)$ & $47.1(36.8-62.2)$ & $43.0(25.5-66.7)$ & $44.5(44.3-50.7)$ & $p=0.87$ \\
Angiopoietin-2 $\left(\mathrm{ng} . \mathrm{ml}^{-1}\right)$ & $4.7(3.5-6.5)$ & $6.8(5.0-12.5)$ & $12.7(6.4-20.5)$ & $p<0.01$ \\
Soluble Tie2 $\left(\mathrm{ng} . \mathrm{ml}^{-1}\right)$ & $11.8(6.9-17.1)$ & $15.3(12.0-20.8)$ & $13.4(8.1-24.6)$ & $p=0.31$ \\
Von Willebrand factor $\left(\mathrm{U} . \mathrm{ml}^{-1}\right)$ & $2.5(1.9-3.2)$ & $2.2(1.4-3.4)$ & $3.3(2.3-6.3)$ & $p=0.17$ \\
\hline
\end{tabular}

ED: emergency department; ICU: intensive care unit; ICU+24h: 24 hours after admission at the ICU. Data are presented as median (IQR) and tested with a Kruskal Wallis test.

tion was found between in vitro endothelial resistance and angiopoietin-1 $(r=-0.57, p=0.15)$, soluble Tie2 $(r=-0.33, p=0.43)$, syndecan-1 $(r=-0.14, p=0.78)$, soluble thrombomodulin $(r=0.21, p=0.66)$ and von Willebrand Factor $(r=-0.40, p=0.33)$ at ED admission, whereas a negative correlation was found between in vitro endothelial resistance and circulating angiopoietin-2 levels $(r=-0.78, p<0.05)$.

\section{Discussion}

In the present study, we showed that plasma from patients following traumatic hemorrhagic shock induced endothelial hyperpermeability, due to disturbed cell-cell integrity, using an in vitro bioassay for endothelial barrier function. This plasma-induced hyperpermeability occurred at an early stage following trauma and hemorrhagic shock, whereas plasma collected at the ICU until $24 \mathrm{~h}$ after admission at the ICU did not induce endothelial hyperpermeability. Interestingly, endothelial hyperpermeability was associated with in vivo microcirculatory perfusion disturbances. These results suggest that in an early stage of traumatic hemorrhagic shock, alterations in plasma induce in vitro endothelial hyperpermeability, but that these changes do not persist at later stages following fluid resuscitation and ongoing treatment of patients.

Current knowledge regarding hemorrhagic shock-induced hyperpermeability is restricted to animal studies [14-18] or in vitro studies investigating the effect of rodent plasma on endothelial barrier 
function [19]. Animal studies showed an early increase in endothelial permeability following hemorrhagic shock [14, 15], which even further increased following fluid resuscitation [16-18]. This was confirmed by a study showing that rodent plasma, collected after fluid resuscitation, induced in vitro rodent endothelial hyperpermeability [19]. However, evidence regarding the effect of human plasma from patients following traumatic hemorrhagic shock on endothelial barrier is limited. Rahbar and colleagues [20] showed that plasma from trauma patients with low colloid osmotic pressure did not affect in vitro endothelial resistance compared to healthy controls, whereas plasma from trauma patients with normal colloid osmotic pressure even increased in vitro endothelial resistance, suggesting solely trauma improves endothelial barrier function. However, these patients did only suffer from trauma without evidence of hemorrhagic shock and plasma was only withdrawn upon arrival at the emergency department. To our knowledge, we are the first to report that plasma obtained from patients following traumatic hemorrhagic shock after admission to the emergency department, but not after admission to the ICU, induced in vitro endothelial hyperpermeability. These results increase our knowledge regarding the impact of traumatic hemorrhagic shock on endothelial permeability and its course following standard treatment.

Standard treatment strategies, consisting of fluid therapy, may impact the magnitude of the observed effect on endothelial barrier function. Crystalloids and noradrenaline are factors known to worsen interstitial edema $[14,21]$, while fresh frozen plasma may protect in vitro endothelial barrier function [14]. We showed that plasma collected at ICU admission and 24 hours later did not affect in vitro endothelial barrier function compared to controls. The absence of endothelial hyperpermeability at these time points could not be explained by hemodilution as we previously showed that hemodilution rather worsens than improves in vitro endothelial barrier function [12]. We previously also showed that plasma from patients undergoing cardiac surgery with cardiopulmonary bypass impaired in vitro endothelial barrier function which continued in the first postoperative days regardless of hemodilution and blood transfusion [22]. Taken together, this suggests that hemorrhagic shock-induced hyperpermeability was transient as plasma obtained after fluid resuscitation and ongoing treatment did not induce in vitro endothelial hyperpermeability.

Microcirculatory perfusion disturbances are present in an early stage in patients following traumatic hemorrhagic shock [3, 4, 9]. However, contrasting results exist regarding the course of restoration following fluid resuscitation $[3,4,9]$. In the present patient population, microcirculatory flow improved following standard treatment $[4,9]$. In accordance, we showed that plasma obtained after standard treatment did not affect in vitro endothelial permeability and that in vitro endothelial resistance was positively associated with in vivo microcirculatory perfusion. Taken together, these results support endothelial hyperpermeability as underlying problem in the development of microcirculatory perfusion disturbances [23].

As a first step in determining a biomarker for endothelial hyperpermeability following traumatic hemorrhagic shock, we studied circulating levels of proteins associated with glycocalyx degradation, endothelial injury and endothelial permeability, including the angiopoietin/Tie 2 system. Animal studies showed that therapeutically targeting the angiopoietin/Tie2 system, a key regulator of the endothelial barrier, reduced microvascular leakage [17, 18] and restored microcirculatory perfusion [17] following hemorrhagic shock and fluid resuscitation. The angiopoietin/Tie 2 system consists of the endothelium specific tyrosine kinase receptor Tie2 with binding sites for angiopoietin-1 and angiopoietin-2 [24]. Angiopoietin-1 maintains endothelial barrier function, whereas angiopoietin-2 is released during stress and induces vascular leakage. Previous studies showed that in patients with traumatic hemorrhagic shock, circulating angiopoietin-2 levels associated with endothelial activation, injury severity and detrimental clinical outcome [25]. Interestingly, we found that circulating angiopoietin-2 levels were negatively associated with endothelial resistance at ED admission. As circulating angiopoietin-2 levels increased over time, it seems that angiopoietin-2 alone is not responsible for the barrier disruptive 
effect. This is in line with a murine study where angiopoietin-2 only induced microvascular leakage in combination with a vasoactive cytokine [26]. Further investigation is warranted to elaborate on the role of angiopoietin-2 in the development of endothelial hyperpermeability following traumatic hemorrhagic shock.

Other studied markers included syndecan-1, von Willebrand factor and soluble thrombomodulin. Shedding of syndecan-1, a marker for endothelial glycocalyx degradation, is found to be associated with increased mortality in patients following hemorrhagic shock [20, 25, 27]. Von Willebrand factor and soluble thrombomodulin are markers reflecting endothelial injury and activation, respectively [28]. In the current setting we did not find a relation between circulating syndecan-1, von Willebrand factor, soluble thrombomodulin and in vitro endothelial hyperpermeability, however, it remains interesting to further elaborate the role of these markers on endothelial hyperpermeability following traumatic hemorrhagic shock.

\subsection{Limitations}

There were several limitations to the present study. First, a relatively small number of patients was included in the present sub-study. Although powered for in vitro endothelial barrier measurements, additional analyses, such as determining a biomarker of interest, were limited and no hard conclusions could be drawn. Interesting is however, that although sample size was small and samples came from a heterogenic population, significant differences were found in endothelial permeability.

Secondly, the majority of the patients included in this sub-study were male, making it difficult to translate the findings to the female population. However, the population of patients with traumatic hemorrhagic shock is known to be predominantly male. Also, animal studies showed that the female gender exhibits microvascular protection [29], suggesting that the effects of plasma from female patients on in vitro endothelial barrier function should be studied separately.

Finally, the effect of plasma was studied on "healthy" endothelial cells in an in vitro setting, which limits translation to the in vivo situation. However, previous studies confirmed the representability of these cells as a model for endothelial barrier function [12, 13, 30].

\section{Conclusion}

We report that early changes in plasma from patients following traumatic hemorrhagic shock induced endothelial hyperpermeability as determined by an in vitro bioassay of endothelial barrier function, which coincided with in vivo microcirculatory perfusion disturbances. Both effects diminished at later stages after fluid resuscitation and ongoing treatment of patients. Future investigations should focus on early alterations in circulating markers to identify potential treatment targets and strategies.

\section{Acknowledgments}

Not applicable.

\section{Funding}

This work was supported by the European Society of Anesthesiology [Research project grant 2016 to C.E.v.d.B.]; the European Society of Intensive Care Medicine [Levi-Montalcini Award 2017 to C.E.v.d.B.]; the Dutch Society of Anesthesiology [Young Investigator Grant 2017 to C.E.v.d.B.]; the 
Dutch Research Council [Veni grant 2019 to C.E.v.d.B]; the Dutch Heart Foundation [2016T064 to N.A.M.D.] and CSL Behring [Professor Heimburger Award 2019 to N.A.M.D].

\section{References}

[1] Mehta D, Malik AB. Signaling mechanisms regulating endothelial permeability. Physiol Rev. 2006;86(1):279-367.

[2] Vincent JL, De Backer D. Circulatory shock. N Engl J Med. 2013;369(18):1726-34.

[3] Tachon G, Harrois A, Tanaka S, Kato H, Huet O, Pottecher J, et al. Microcirculatory alterations in traumatic hemorrhagic shock. Crit Care Med. 2014;42(6):1433-41.

[4] Hutchings SD, Naumann DN, Hopkins P, Mellis C, Riozzi O, Sartini S, et al. Microcirculatory impairment is associated with multiple organ dysfunction following traumatic haemorrhagic shock: The MICROSHOCK study. Crit Care Med. 2018;46(9):e889-96.

[5] Kauvar DS, Lefering R, Wade CE. Impact of hemorrhage on trauma outcome: An overview of epidemiology, clinical presentations, and therapeutic considerations. J Trauma. 2006;60(6 Suppl):S3-11.

[6] Gulati A. Vascular Endothelium and Hypovolemic Shock. Curr Vasc Pharmacol. 2016;14(2):187-95.

[7] Torres Filho I. Hemorrhagic Shock and the Microvasculature. Compr Physiol. 2017;8(1):61-101.

[8] Hutchings SD, Naumann DN, Harris T, Wendon J, Midwinter MJ. Observational study of the effects of traumatic injury, haemorrhagic shock and resuscitation on the microcirculation: A protocol for the MICROSHOCK study. BMJ Open. 2016;6(3):e010893.

[9] Naumann DN, Hazeldine J, Midwinter MJ, Hutchings SD, Harrison P. Poor microcirculatory flow dynamics are associated with endothelial cell damage and glycocalyx shedding after traumatic hemorrhagic shock. J Trauma Acute Care Surg. 2018;84(1):81-8.

[10] Naumann DN, Hazeldine J, Dinsdale RJ, Bishop JR, Midwinter MJ, Harrison P, et al. Endotheliopathy is associated with higher levels of cell-free DNA following major trauma: A prospective observational study. PLoS One. 2017;12(12):e0189870.

[11] Langeler EG, van Hinsbergh VW. Characterization of an in vitro model to study the permeability of human arterial endothelial cell monolayers. Thromb Haemost. 1988;60(2):240-6.

[12] Koning NJ, Overmars MA, van den Brom CE, van Bezu J, Simon LE, Vonk AB, et al. Endothelial hyperpermeability after cardiac surgery with cardiopulmonary bypass as assessed using an in vitro bioassay for endothelial barrier function. Br J Anaesth. 2016;116(2):223-32.

[13] Szulcek R, Bogaard HJ, van Nieuw Amerongen GP. Electric cell-substrate impedance sensing for the quantification of endothelial proliferation, barrier function, and motility. J Vis Exp 2014;28:85.

[14] Peng Z, Pati S, Potter D, Brown R, Holcomb JB, Grill R, et al. Fresh frozen plasma lessens pulmonary endothelial inflammation and hyperpermeability after hemorrhagic shock and is associated with loss of syndecan 1 . Shock. 2013;40(3):195-202.

[15] Potter DR, Miyazawa BY, Gibb SL, Deng X, Togaratti PP, Croze RH, et al. Mesenchymal stem cell-derived extracellular vesicles attenuate pulmonary vascular permeability and lung injury induced by hemorrhagic shock and trauma. J Trauma Acute Care Surg. 2018;84(2):245-56.

[16] Childs EW, Udobi KF, Hunter FA, Dhevan V. Evidence of transcellular albumin transport after hemorrhagic shock. Shock. 2005;23(6):565-70.

[17] Trieu M, van Meurs M, van Leeuwen AL, Van Slyke PV, Hoang V, Geeraedts LM, et al. Vasculotide, an angiopoietin-1 mimetic, restores microcirculatory perfusion and microvascular leakage and decreases fluid resuscitation requirements in hemorrhagic shock. Anesthesiology. 2018;128(2):361-74.

[18] Childs EW, Tharakan B, Byrge N, Tinsley JH, Hunter FA, Smythe WR. Angiopoietin-1 inhibits intrinsic apoptotic signaling and vascular hyperpermeability following hemorrhagic shock. Am J Physiol Heart Circ Physiol. 2008;294(5):H2285-95.

[19] Sawant DA, Tharakan B, Tobin RP, Stagg HW, Hunter FA, Newell MK, et al. Inhibition of Fas-Fas ligand interaction attenuates microvascular hyperpermeability following hemorrhagic shock. Shock. 2013;39(2):161-7.

[20] Rahbar E, Cardenas JC, Baimukanova G, Usadi B, Bruhn R, Pati S, et al. Endothelial glycocalyx shedding and vascular permeability in severely injured trauma patients. J Transl Med. 2015;13:117.

[21] Farand P, Hamel M, Lauzier F, Plante GE, Lesur O. Review article: Organ perfusion/permeability-related effects of norepinephrine and vasopressin in sepsis. Can J Anaesth. 2006;53(9):934-46.

[22] Dekker NA, van Leeuwen AL, van Strien WW, Majolée J, Szulcek R, Vonk AB, et al. Microcirculatory perfusion disturbances following cardiac surgery with cardiopulmonary bypass are associated with in vitro endothelial hyperpermeability and increased angiopoietin-2 levels. Crit Care. 2019;23:117. 
[23] Duan CY, Zhang J, Wu HL, Li T, Liu LM. Regulatory mechanisms, prophylaxis and treatment of vascular leakage following severe trauma and shock. Mil Med Res. 2017;4:11.

[24] Parikh SM. Angiopoietins and Tie2 in vascular inflammation. Curr Opin Hematol. 2017;24(5):432-38.

[25] Halbgebauer R, Braun CK, Denk S, Mayer B, Cinelli P, Radermacher P, et al. Hemorrhagic shock drives glycocalyx, barrier and organ dysfunction early after polytrauma. J Crit Care. 2018;44:229-37.

[26] Benest AV, Kruse K, Savant S, Thomas M, Laib AM, Loos EK, et al. Angiopoietin-2 is critical for cytokine-induced vascular leakage. PLoS One. 2013;8(8):e70459.

[27] Johansson PI, Stensballe J, Rasmussen LS, Ostrowski SR. A high admission syndecan-1 level, a marker of endothelial glycocalyx degradation, is associated with inflammation, protein $\mathrm{C}$ depletion, fibrinolysis, and increased mortality in trauma patients. Ann Surg. 2011;254(2):194-200.

[28] Aird WC. Endothelium as an organ system. Crit Care Med. 2004;32(5 Suppl):S271-79.

[29] Panazzolo DG, Silva LH, Cyrino FZ, Sicuro FL, Kraemer-Aguiar LG, Bouskela E. Gender differences in microcirculation: Observation using the hamster cheek pouch. Clinics (Sao Paulo). 2013 Dec;68(12):1537-42.

[30] Klein CL, Köhler H, Bittinger F, Wagner M, Hermanns I, Grant K, et al. Comparative studies on vascular endothelium in vitro. Pathobiology. 1994;62:199-208. 